\title{
XXVI.
}

Aus der inneren Abteilung des städtischen Krankenhauses in Augsburg (Oberarzt Dr. L. R. Müller).

\section{Über einen Fall von syphilitischer Spinalparalyse.}

\author{
Von \\ Dr. Renner, \\ Assistenzarzt. \\ (Mit Tafel III.)
}

Das 1892 von Erb aufgestellte Krankheitsbild der syphilitischen Spinalparalyse hat seitdem durch eine Reihe von Beiträgen an Daseinsberechtigung gewonnen. 1903 unterzog Erb ${ }^{1}$ ) noch einmal alle bis dahin mitgeteilten Fälle einer kritischen Revision; in der Folgezeit konnten wiederholt $\mathrm{Nonne}^{2}$ ) und $\mathrm{Wimmer}{ }^{3}$ ) wichtige Beiträge zu dieser Frage liefern. Die nicht sehr zahlreiche Kasuistik sind wir in der Lage um einen weiteren interessanten Fall zu vermehren, der von den bisher mitgeteilten einige Abweichungen bietet.

Es handelt sich um den 36 jährigen Maschinenarbeiter S., der am 28. III. 1907 in dàs städtische Krankenhaus zu Augsburg aufgenommen wurde und am 19. IV. daselbst starb.

Vorgeschichte: S. stammt aus gesunder Familie, ist kinderlos verheiratet; seine Frau hatte 2 Frühgeburten. Vor 9 Jahren wurde ihm angeblich wegen Wasserbruches der linke Hoden operativ entfernt. Schon seit einigen Jahren hat P. über vorübergehende Schmerzen in beiden Beinen zu' klagen. Ferner leidet er schon seit einiger Zeit an Wadenkrämpfen. Seit Dezember 1906 haben sich die Schmerzen und Krämpfe gesteigert: "es zog ihm manchmal die Beine herauf gegen den Leib". Während ber Schmerzanfälle war P. unsicher auf den Beinen, sonst bestanden keine stärkeren Gehstörungen. Seit 13 Wochen ist er arbeitsunfähig und in ärztlicher Behandlung. Eine am 1. Februar auf Anraten des behandelnden Arztes begonnene Schmierkur brachte keine Besserung. Es trat nun eine derartige Schwäche in den Beinen dazu, dass er sich nicht mehr allein auf den Füssen halten konnte. Doch konnte er im Liegen noch alle Bewegungen mit den Extremitäten ausführen. Vor 7 Wochen machten sich

1) Bd. 23 dieser Zeitschrift.

2) Bd. 29 u. 30 dies. Zeitschr.

3) Bd. 32 dies. Zeitschr. 
zum ersten Male Störungen in der Harnentleerung bemerkbar. P. konnte damals plötzlich keinen Urin mehr lassen und musste vom Arzt katheterisiert werden. In der nächsten Zeit floss dann der Urin in Tropfen $a b$, in letzter Zeit in Zwischenräumen und grösseren Portionen.

Stuhlbeschwerden waren nie vorhanden. Seit 3 Jahren soll die Sehkraft des linken Auges abgenommen haben. Doppeltsehen bestand nie. Sexuelle Infektion wird geleugnet, mässiges Potatorium zugegeben.

Status praesens: Mittelgrosser, kräftig gebauter und gesund aussehender Mann. P. liegt mit angezogenen Beinen im Bett. Die linke Pupille ist weiter wie die rechte, beide sind reflektorisch lichtstarr, Accomodation erbalten. Keine Augenmuskelstörungen. Spiegelbefund: Links grauweissliche, deutlich exkavierte (atrophische) Papille, rechts normale Verhältnisse. Keine Sprachstörungen; auch die übrigen Gehirnnerven frei.

Lungenbefund wie Herzbefund ohne Besonderheiten. Puls 100. Am Präputium und Glans penis Exkoriationen.

Die aktive Beweglichkeit ist in allen Gelenken erhaiten; doch gehen nach aktivem Strecken die Beine allmählich wieder in die gebeugte Stellung zurück. Zielbewegungen mit den Fingern werden unsicher ausgeführt, dagegen gelingt der Kniehackenversuch gut. Bei Prüfung der Sensibilität ergibt sich eine hypästhetische Zone von den Brustwarzen abwärts bis handbreit innerhalb dos Nabels, hinten rom unteren Scapularwinkel bis zum 1. Lendenwirbel. Soust bestehen keine Sensibilitätsstörungen.

Reflexe: Radiusperiostreflexe ebenso Tricepssehnenreflexe lebhaft, Bauchdeckenreflex links angedeutet, rechts + . Patellarreflexe lebhaft, lebhafter Fussklonus. Babinskisches Phänomen beiderseits positiv.

Incontinentia urinae. Im Urin reichlicher Bodensatz von Eiterzellen. Reaktion alkalisch, stechender Geruch.

Während der ersten 10 Tage des Krankenhausaufenthaltes traten anfallsweise heftige krampfartige Sehmerzen in der Urethra auf, die durch sofortiges Katheterisieren sich günstig beeinflussen liessen: sie erinnerten an tabische Urethralkrisen. Die Abendtemperaturen waren ständig erhöht, einmal bis 39,6 . Der Harnbefund liess diese Fiebererscheinungen leicht als Pyelitis und Pyelonephritis erklären. Während in der ersten Zeit das Sensorium vollständig klar war, halluzinierte der Kranke später nachts und war sehr unruhig, so dass seine Verlegung auf die psychiatrische Abteilung notwendig wurde. Es traten auch starke Diarrhöen auf. Beide Erscheinungen mussten bei dem zunehmenden Kräfteverfall auf Intoxikation von seiten der Harnwege zurückgeführt werden. Die nervösen Störungen blieben während des Krankheitsverlaufes die gleichen. Eine in den letzten Tagen vor dem Tode vorgenommene Lumbalpunktion zeigte eine deutliche Vermehrung der Lymphocyten in der Spinalflüssigkeit. Nachdem P. zuletzt noch vollständig somnolent geworden war, trat am 22. Tage seines Krankenhausaufenthaltes der Exitus ein.

Die Diagnose lautete: Kombinierte Systemerkrankung auf luetischer Basis, Cystitis, aufsteigende Pyelitis und Pyelonephritis.

Die Sektion konnte mit folgendem Befunde unsere Diagnose bestätigen:

Luetische Endaortitis, Cystitis, Pyelitis, Pyelonephritis, septischer Milztumor.

Gehirn: Hirnhäute nicht getrübt, Windungen nicht wesentlich abgeflacht, auf Frontalschnitten makroskropisch kein Befund. 
Rückenmark dünn, nirgends eine Auftreibung oder Einschnürung, Häute nicht verdickt. Das Rückenmark wird in toto gehärtet. Die mikroskopische Untersuchung der nach Weigert, van Gieson und mit Hämatoxylin-Eosin gefärbten Schnitte bringt folgendes Ergebnis:

Oberstes Sakralmark (Fig. 1, Taf. III). Im Gebiet der Seitenstränge zeigt sich ein an der Peripherie gelegenes kleines dreieckiges Feld aufgehellt. Es entspricht der Lage der PyS. Alle übrigen Felder sowie auch die graue Substanz sind völlig intakt.

Mittleres Lumbalmark (Fig. 2, Taf. III). Das erwähnte Degenerationsgebiet der PyS tritt auch hier deutlich hervor and reicht nicht mehr völlig bis zur Peripherie. Der übrige Querschnitt bietet normale Verhältnisse.

Im Übergangsteil vom Lumbal-zum Dorsalmark (Fig. 3, Taf. III). findet sich ebenfalls eine deutliche Aufhellung der PyS, die hier an Ausdehnung zugenommen haben.

Im oberen Dorsalmark (Fig. 4, Taf. III) ist neben der Degeneration in den $\mathrm{PyS}$ eine eben erkannbare, ganz geringe Lichtung der Burdachschen. Stränge festzustellen.

Diese wird deutlicher im untersten Halsmark (Fig. 5, Taf. III); and zwar sind namentlich die mittleren Partien der Keilstränge befallen, während die hinteren und vorderen seitlichen Felder fast ganz verschont geblieben sind. Daneben tritt auch in dieser Höhe eine ausgedehnte, aberetwas weniger intensive Aufhellung in den PyS zutage.

Im mittleren Halsmark (Fig. 6, Taf. III) ist keine wesentliche Veränderung aufgetreten. Von den Hintersträngen sehen wir die Gol]schen Stränge völlig unversehrt, die Burdachschen in der vorher beschriebenen Weise, nur auf einer Seite vielleicht etwas stärker angegriffen. In den PyS macht sich auch hier nur eine ganz geringe Aufhellung bemerkbar.

Im obersten Halsmark (Fig. 7, Taf. III) treffen wir auf ein kommaartiges, jetzt dentlicher hervortretendes Degenerationfeld, das sich durch einen schmalen, gut erhaltenen Streifen von den Hinterhörmern abgrenzen lässt. Auch die vordersten Gebiete der Gollschen Strän̆ge sind leicht angegriffen. In den PyS ist noch eine ganz geringe Lichtung nachzuweisen.

Ein Schnitt durch den Beginn der Pyramidenkreuzung zeigt in. den Hintersträngen das eben beschriebene Bild, während sich an den Pyramidenbahnen keine Veränderung mehr erkennen lässt.

Fassen wir Krankengeschichte und Sektionsbefund noch einmal kurz zusammen:

Ein 36jäbriger Mann, dessen syphilitische Infektion aus der Anamnese vermutungsweise hervorgeht, hat schon einige Jahre vor seiner eigentlichen Erkrankung anfallsweise an Schmerzen in den Beinen und Wadenkrümpfen gelitten. 4 Monate ror seinem Tode treten deutlich spastische Erscheinungen zutage. Daneben stellt sich Unsicherheit beim Gehen ein. Eine Schmierkur bringt keine Besserung, das Gehen wird vielmehr bald ganz unmöglich. 7 Wochen vor dem Tode treten Blaisenstörungen auf. Bei Krankenhauseintritt sind deutliche Zeichen einer Cystitis vorhanden. Reflektorische Pupillenstarre, Opticusatrophie, Ataxie 
in den Armen, leichte gürtelförmige Sensibilitätsdefekte und Blasenstörungen lassen an eine Tabes denken, dagegen dokumentieren spastischparetische Erscheinungen in den unteren Extremitäten, die lebhaften Sebnenreflexe, das Vorhandensein des Babinskischen Phänomens, dass die PyS ergriffen sind. Psychische Störungen machen sich erst gegen das Ende des Lebens bemerkbar; sie können wohl nur als Intoxikationserscheinungen infolge der aufsteigenden Blaseninfektion gedeutet werden.

Die Sektion bestätigt unsere auf "kombinierte Systemerkrankung syphilitischer Natur" gestellte Diagnose. Die Veränderungen im Halsmark entsprechen einer beginnenden Tabes superior. Sie betreffen vorwiegend die Burdachschen Stränge, sind andeutungsweise schon im oberen Brustmark zu treffen und nehmen dann bis zur grössten Intensität im obersten Halsmark ständig zu. Daneben ist schon im oberen Halsmark eine PyS-Degeneration sichtbar, deren Faserschwund nach unten zu deutlich stärker wird. Eine nennenswerte Verdickung der Pia, irgend ein lokal meningitischer Herd oder eine Kompressionsstelle lässt sich nach makroskopischem wie mikroskopischem Befunde mit Sicherheit ausschliessen.

Das klinische Bild dieser Erkrankung findet somit restlos in dem anatomischem Befunde seine Erklärung. Die Ataxie in den Armen entspricht der beschriebenen Sklerose in den Keilsträngen des Halsmarks. Auch die reflektorische Pupillenstarre lässt sich, wenn man den Ansichten Reichardts folgt, auf Kosten dieser Hinterstrangdegeneration setzen. Im Brust- und Lendenmark sind die Hinterstränge intakt; es liess sich auch am unteren Rumpfe und den Beinen keine Ataxie feststellen. Der Degeneration der Pyramidenseitenstränge, welche an Intensität von oben nach unten zanimmt, entsprechen die Steigerung der Reflexe, die Spasmen und die Paresen in den unteren Extremitäten.

Wir haben also klinischeine Kombination von beginnender Halsmarktabes mit einer ausgesprochenen spastischen Spinalparalyse, anatomisch eine kombinierte Strangdegeneration, die im Halsmark einen Teil der Hinterstränge und die Pyramidenseitenstrangbahn, im übrigen Rückenmark ausschliesslich die Pyramidenseitenstrangbahn betrifft.

Ihrer Ätiologie nach ist die Erkrankung zweifellos syphilitischer Natur. Wenn auch die luetische Infektion vom Patienten selbst nicht zugegeben wird, so ist sie doch aus den übrigen Angaben der Anamnese mit "Sicherheit anzunehmen. Andere metasyphilitische Veränderungen (Opticusatrophie, reflektorische Pupillenstarre und Vermehrung der Lymphocyten in der Lumbalflüssigkeit) sprechen in diesem Sinne. "Kombinierte Systemerkrankung auf luetischer Basis" hatte unsere 
Diagnose gelautet. Die Diagnose lässt sich aber noch präziser fassen, wenn wir das eingangs erwähnte, von Erb eingeführte Krankheitsbild der syphilitischen Spinalparalyse berücksichtigen. Der gleiche Symptomenkomplex wie dort beherrscht auch unseren Krankheitsfall: Neben den Erscheinungen der spastischen Spinalparalyse finden sich Störungen in der Blasenfunktion und in der Sensibilität. Nur die Forderungen: Freibleiben der Hirnnerven und Fehlen von Pupillenstörungen, sind in unserem Falle nicht erfüllt. Doch glaube ich hierauf ebenso wie Nonne weniger Gewicht legen zu müssen. Da die syphilitische Ätiologie zu den Grundbedingungen dieser Krankheitsform gehört, wird man sich nicht wundern dürfen, wenn hier und da auch andere metasyphilitische Erscheinungen zutage treten.

Die vorliegende Beobachtung unterscheidet sich also insofern von den bisher mitgeteilten Fällen der syphilitischen Spinalparalyse, als sich zu den spastisch-paretischen Erscheinungen Ataxie in den oberen Extremitäten, zu der Degeneration der Pyramidenseitenstränge eine solche in den Burdachschen Bündeln hinzugesellte. Auch das relativ schnelle Fortschreiten der nervösen Symptome zeichnet ihn ror anderen Fällen aus. 
Fig. l.

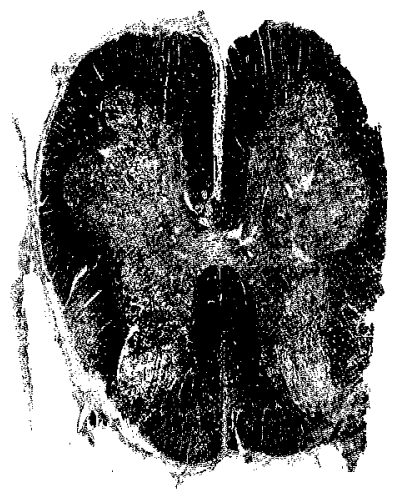

Fig.?
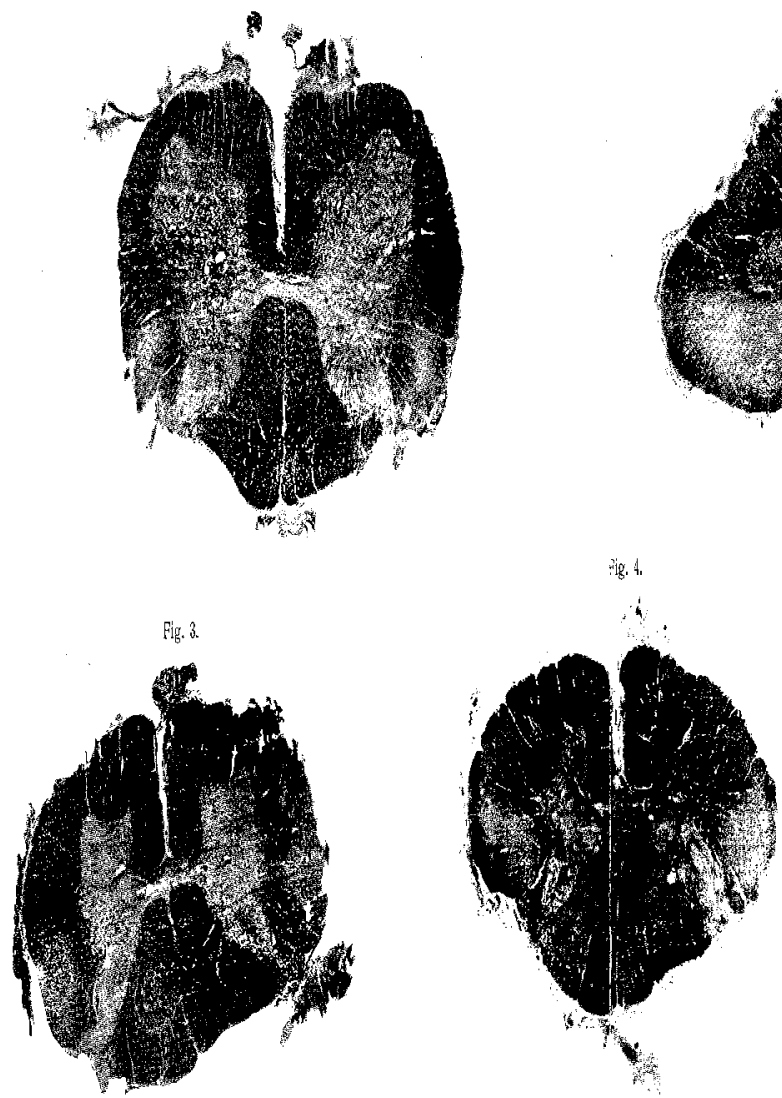

Fig, 5 .

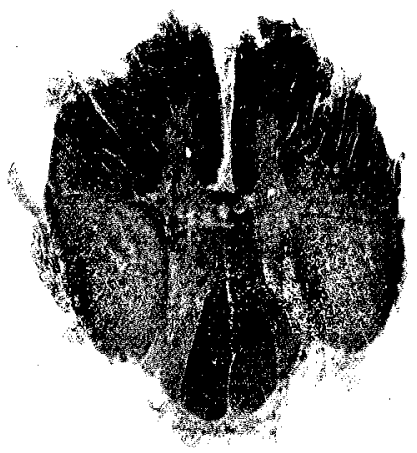

Fig. 6.

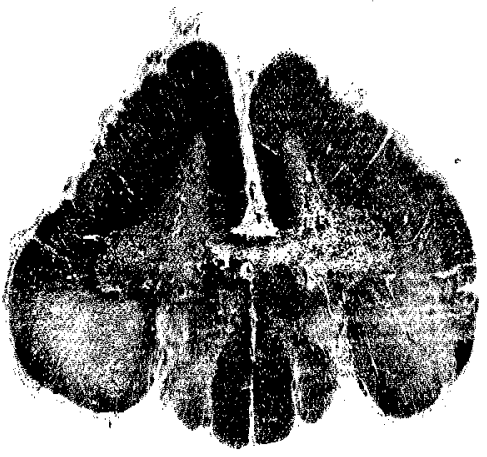

Fig. . .

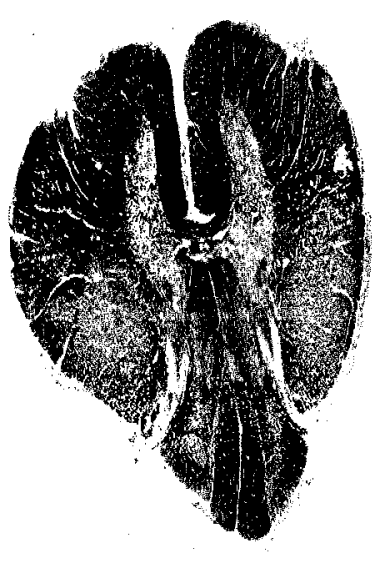

